\title{
Firm and User Generated Content in the Digital Economy: Key Players, Management and Impact
}

\author{
Minitrack Chair \\ Michael Klaas \\ Zurich University of Applied \\ Sciences \\ Michael.klaas@zhaw.ch
}

\author{
Minitrack Co-Chair \\ Bettina Beurer-Zuellig \\ Zurich University of Applied \\ Sciences \\ Bettina.beurer-zuellig@zhaw.ch
}

\section{Minitrack Description}

The Minitrack Content (FGC/UGC) in the Digital Economy takes into account the communication shift from traditional one-way to two-way communication, with content being a means to earn trust with consumers. Content creation by companies is referred to as firm-generated content (FGC) or producergenerated content (PGC). User-generated content (UGC) also termed consumer-generated content (CGC) is voluntarily "created by the general public" and does not necessarily follow financial interests (Arnhold 2010, p. 33). Consumers consult UGC and FCG as a source of inspiration when making purchasing decisions. Source criteria, media format, valence as well as depth and breadth impact the helpfulness and credibility of content. From a business perspective UGC plays an important role in the customer journey, influencing purchasing decisions, raising brand awareness, brand loyalty as well as perceived quality.

\section{Papers in Minitrack}

\section{Consumers' Need for Negative Product Information Before Reading Reviews \\ Stefan Hirschmeier, Roman Tilly}

Negative product-related information is crucial to consumers in purchase decisions. Consumers perceive negative information stronger than positive, and next to a stronger perception, consumers also have a high demand for negative product aspects, as these show the problem areas of a product and can help avoid losses. But negative product-related information is not available in the product search process until the customer reads reviews at a very late phase of the decision process. Even though we know about a bias in perception of negative information, little is known about the exact need for negative product-related information during the search process. We examine the need for negative productrelated information throughout the purchase-decision process for different product types. Insights about the need for negative product-related information can inform ecommerce platform providers how to design a better product search on their site.

\section{When More is More and Less is More: Depth and Breadth of Product Reviews and Their Effects on Review Helpfulness}

Jaebong Son, Arash Negahban, Youngsu Lee, James Connolly, Dalen Chiang

With the growth of e-commerce coupled with mobile technology, more online shoppers rely on fellow consumers' reviews to better diagnose products as a means to decrease product uncertainty. Comprehending what factors of reviews are considered helpful for product diagnosticity becomes imperative. One significant factor is the depth of information conveyed in reviews, and we add a complementary measure, called review breadth. Based on the statistical and predictive analyses, we examine the relationship between review breadth and review helpfulness. The empirical findings claim that review breadth has a significant effect on the helpfulness of reviews over and above review depth. This study makes contributions to the extant literature of product reviews and e-commerce. As an explanatory variable, review breadth is statistically significant to capture a review's information along with review depth. As a predictor variable, it improves classifiers' prediction accuracy of whether reviews are considered to be helpful. 


\section{Perceived Firm Attributes, Social Identification, and Intrinsic Motivation to Voice in Brand Virtual Communities: Differentiating Brand-General and Innovation-Specific Perceptions \\ Yongqiang Sun, Cailian Zhao, Xiao-Liang Shen, Nan Wang}

The question about why some brand virtual communities (BVCs) successfully motivate customers to engage in value creation (e.g., voice) while others do not is still an important but understudied research issue. To fill this research gap, we propose a research model to shed light on the antecedents of intrinsic motivation to voice by focusing on the role of perceived firm attributes. Specifically, we argue that firm attributes can be classified into brand general versus innovationspecific attributes which affect intrinsic motivation through two types of social identification namely brand identification and community identification respectively. The links between these two types of perceptions are examined too. A field study of 291 BVC users was conducted to test the research model. The results show that customer orientation and perceived openness positively affect customers' brand identification and community identification respectively, and customer orientation has a positive effect on perceived openness. Furthermore, the impact of brand identification on intrinsic motivation is found to be fully mediated by community identification 